\title{
O BACHARELADO INTERDISCIPLINAR EM SAÚDE E MEDICINA: UMA ANÁLISE DE CORRESPONDÊNCIA DE CONCEPÇÕES ACERCA DE ATENDIMENTO HUMANIZADO
}

\author{
Renata Meira Veras ${ }^{1}$, Vitória Batista Calmon de Passos ${ }^{1}$, Caio Cézar Moura Feitosa ${ }^{1}$ \\ e Sheyla Fernandes ${ }^{2}$ \\ ${ }^{1}$ Universidade Federal da Bahia, Brasil.renatameiraveras@gmail.com; vitoria.passos17@gmail.com \\ feitosacaiocezar@gmail.com. \\ ${ }^{2}$ Universidade Federal de Alagoas, Brasil. sheyla.fernandes@ip.ufal.br
}

\begin{abstract}
Resumo. Introdução: Uma medicina exercida com excelência deve ser baseada tanto em sua qualidade técnica quanto em seu caráter humano. Nesse cenário, o Bacharelado Interdisciplinar em Saúde (BIS) surgiu na intenção de adaptar a formação em saúde às mudanças geradas em decorrência do esgotamento do modelo de ensino vigente, oferecendo uma formação multi e interdisciplinares e humanista. Objetivo: Esse artigo tem como objetivo analisar as concepções de atendimento médico humanizado dos estudantes de medicina, identificando possíveis diferenças entre os discursos dos estudantes que cursaram o BIS e os que não cursaram. Método: Trata-se de um estudo qualitativo, empírico e exploratório que analisa as concepções utilizando suporte do Software Iramuteq para processamento dos dados. As informações utilizadas foram coletadas mediante aplicação de questionário com questões abertas a 344 estudantes de medicina matriculados entre o primeiro e oitavo semestres. Resultados: A análise de correspondência indicou que os estudantes que cursaram o primeiro ciclo tendem a atrelar seus discursos acerca do atendimento médico humanizado ao conceito ampliado de saúde, enfatizando os determinantes sociais da saúde como fundamentais para o desenvolvimento da relação humanizada. Conclusões: A formação interdisciplinar pode influenciar uma formação mais humanizada e coerente com a realidade no campo da saúde pública.
\end{abstract}

Palavras-chave: Educação Médica; Humanização da Assistência; Universidade; Currículo; Interdisciplinaridade

THE INTERDISCIPLINARY BACHELOR IN HEALTH AND MEDICINE: A CORRESPONDENCE ANALYSIS OF CONCEPTIONS ABOUT HUMANIZED CARE

Abstract. Introduction: A medicine practiced with excellence must be based on both its technical quality and its human character. In this scenario, the Interdisciplinary Bachelor of Health (BIS) emerged with the intention of adapting health education to the changes generated as a result of the exhaustion of the current teaching model, offering multi and interdisciplinary and humanistic training. Goals: This article aims to analyze the medical students' conceptions of humanized medical care, as well as to identify possible differences between the discourses of students who attended the BIS and those who did not attend. Methods: It is a qualitative, empirical and exploratory study that analyzes the concepts through the Iramuteq Software. The data used were collected by applying a questionnaire with open questions to 344 medical students enrolled between the first and eighth semesters. Results: Correspondence analysis indicated that students who attended the first cycle tend to tie their discourses about humanized medical care to the expanded concept of health, emphasizing the social determinants of health as fundamental to the development of the humanized relationship. Conclusions: Thus, it is concluded that the interdisciplinary trainee allows for a more humanized training and consistent with the reality in the field of public health.

Keywords: Medical Education; Humanization of Assistance; University; Curriculum; Interdisciplinarity 


\section{INTRODUÇÃO}

A Política Nacional de Humanização (PNH) foi criada no Brasil com o objetivo de melhorar a qualidade dos serviços prestados no campo da saúde, articulando os avanços tecnológicos e o desenvolvimento de ações nos eixos da Formação de profissionais, Gestão dos serviços e Atendimento oferecido aos usuários dos SUS (Brasil, 2004). Sendo assim, a relação médico-paciente torna-se o principal elemento a ser considerado na sua implantação (Rios \& Sirino, 2015).

O conceito de humanização da assistência a ser considerado nesse estudo é concebido a partir de uma maior aproximação com a Saúde Coletiva, de modo que, mesmo sem dispor de uma única definição teórica para o mesmo, o sentido que ele comumente assume é de um conjunto de ações relacionadas à atenção em saúde, que contribuam para promoção da articulação entre aspectos técnicos, com outros subjetivos, culturais, relacionais, de profissionais e usuários dos serviços de saúde (Deslandes, 2006). Diante disso, dando enfoque à formação médica orientada pela ideia de humanização, e considerando a polissemia desse conceito, é interessante que essa formação seja pensada compreendendo-se como seu componente norteador a Política Nacional de Humanização. Uma formação médica humanística, portanto, compreende a "[...] aquisição processual de conhecimentos específicos da área de Humanidades, nesse escopo mais amplo e particularmente destacando a conscientização do comportamento moral e o desenvolvimento de habilidades de comunicação e construção de vínculos." (Rios \& Schraiber, 2012, p. 12). Seria um tipo de formação em que os cursos considerassem também aspectos subjetivos envolvidos na construção da identidade profissional dos médicos, visto que existem fatores históricos, culturais, interativos, que reforçam a valorização do modelo técnico-científico de assistência. (Traverso-Yépez \& Morais, 2004; Rios \& Schraiber, 2012)

Considerando isso, sabe-se que desde a década de 50 estão presentes no meio acadêmico o debate acerca do esgotamento biomédico e a necessidade de incorporar conhecimentos humanísticos na formação do bacharel em medicina. Mas é a partir da década de 90 que essa discussão surge renovada pela necessidade de reorganizar a formação de graduação em medicina no país com a criação da Comissão Interinstitucional de Avaliação do Ensino Médico (CINAEM) e a publicação das Diretrizes Curriculares Nacionais (DCN) em 2001, revisadas em 2014 (Barros, 2014). No entanto, para além de pensar reformas nos cursos de 
medicina, existem modelos formativos que oferecem opção de formação superior diversificada e inovadora, propiciando a interlocução entre diversos campos do conhecimento (Veras, Coelho, Teixeira \& Traverso-Yépez, 2018; Almeida Filho et al., 2015).

O Bacharelado Interdisciplinar em Saúde (BIS), nesse contexto, foi criado com vistas a repensar a formação de pessoal em saúde, consoante com os princípios do sistema público de saúde vigente no Brasil: a Universalidade do acesso, Equidade na atenção e a Integralidade no atendimento. Esses princípios vão ao encontro do modelo de formação em saúde vigente (e ainda predominante) nesse país que privilegia a especialização precoce, a fragmentação do processo de trabalho e, principalmente, a perda da dimensão humanística no cuidado aos indivíduos (Feuerwerker, 2002).

Assim, o BIS da Universidade Federal da Bahia (UFBA) é um curso de graduação estruturado no modelo de ciclos. Por corresponder ao primeiro ciclo, um dos seus objetivos é a preparação para o ingresso em cursos profissionais, chamados de Cursos de Progressão Linear (CPL); para a formação acadêmica de pós-graduação; ou ainda, para o ingresso no mercado de trabalho em ocupações que não exijam formação profissionalizante (Rocha et al., 2014). Esse modelo de ciclos foi criado na UFBA como uma janela de oportunidade, numa conjuntura política favorável à educação superior pública a partir do Programa de Apoio a Planos de Reestruturação e Expansão das Universidades Federais (REUNI) em 2009, com carga horária de 2400 horas e duração mínima de seis semestres. Dentre os objetivos do REUNI, um deles era "atualizar currículos e projetos acadêmicos visando flexibilizar e melhorar a qualidade da educação superior, bem como proporcionar aos estudantes formação multi e interdisciplinares, humanista e o desenvolvimento do espírito crítico" (Brasil, 2007, p. 9).

Desta maneira espera-se que os egressos do BIS levem as bases humanistas e críticas acerca das necessidades de saúde da população para os diferentes cursos profissionais da UFBA. Torna-se então importante acompanhar quais as contribuições construídas no BIS os estudantes apontam como relevantes em sua formação profissional. Atualmente, existem 291 estudantes egressos do BIS que estão cursando medicina como CPL, dos quais 252 estão matriculados no campus de Salvador e 39 no de Vitória da Conquista (IHAC, 2019). Diante disso, esse estudo tem como questão norteadora: De que maneira os estudantes de medicina egressos do Bacharelado Interdisciplinar em Saúde concebem o que é atendimento médico humanizado em relação aos demais estudantes do curso? Assim, tem- 
se como objetivo analisar se o Bacharelado Interdisciplinar em Saúde contribui para concepções diferenciadas dos estudantes de medicina em comparação aos estudantes optantes pela entrada direta pelo CPL. Especificamente nesse estudo, o enfoque será dado para as concepções acerca do atendimento médico humanizado.

\section{METODOLOGIA}

Trata-se de um estudo transversal, com abordagem qualitativa, no intuito de discutir a influência da graduação prévia no Bacharelado Interdisciplinar em Saúde nas concepções de atendimento humanizado de estudantes de medicina da Universidade Federal da Bahia. Para tanto, desenvolveu-se uma análise fatorial de correspondência apoiada pelo uso do software Iramuteq para o tratamento dos dados. Esse trabalho trata-se de um recorte de uma pesquisa maior intitulada: "O que acham os estudantes de medicina acerca do eixo ético-humanístico da Faculdade de Medicina da Bahia?".

A pesquisa qualitativa permite o estudo de aspectos e fatores da vida humana e a análise dos processos sociais ao longo do tempo. Ela é bastante apropriada para a investigação de problemas/questões pouco conhecidas e que necessitam de compreensão, o que a torna fundamental no presente processo investigativo (Kerr e Kendall, 2013).

\subsection{Cenário e participantes}

$\mathrm{Na}$ Universidade Federal da Bahia ocorreu uma mudança curricular na graduação em medicina no ano de 2007 e, segundo Nery e colaboradores (2013), essa transformação objetivou aperfeiçoar a formação dos médicos buscando adequar-se com o perfil esperado na atualidade. O novo currículo foi organizado por módulos, nos quais a arquitetura curricular buscou contemplar as dimensões técnico-científica, ético-humanística, e de formação em pesquisa. Segundo o Projeto Político Pedagógico do Curso de Graduação em Medicina da FAMEB/UFBA, o eixo ético--humanístico é organizado em oito disciplinas ofertadas entre o primeiro e oitavo semestres e compreende o debate acerca de conhecimentos múltiplos do campo da ética e das humanidades, tendo em vista o desenvolvimento de atitudes éticas e humanas, integrando todas as práticas curriculares, por estar presente em todos os módulos da graduação. (Formigli, 2010).

Existiam, em agosto de 2018, 1023 estudantes matriculados no curso de medicina. Destes, 718 estavam cursando o eixo ético-humanístico ( $1^{\circ}$ ao $8^{\circ}$ semestre), segundo a Faculdade 
de Medicina da Bahia - UFBA. A partir disso, os sujeitos que compuseram essa pesquisa foram selecionados desse universo por adesão voluntária a um questionário aplicado em sala nos horários de algumas aulas com autorização dos docentes e mediante assinatura do Termo de Consentimento Livre e Esclarecido, que Ihes garantiu o anonimato quanto às respostas, mas que autorizou as citações delas, no decorrer da investigação. Vale ressaltar que o estudo foi aprovado pelo Comitê de Ética em Pesquisa da Escola de Enfermagem da UFBA (CAAE: 87862917.8.0000.5531 - Parecer n.2.769.003).

Após a aplicação foram selecionados os questionários respondidos completamente, totalizando 344 estudantes. Destes, foi identificado que 171 não possuíam nenhuma graduação anterior e 107 possuíam alguma graduação anterior, sendo 66 egressos do Bacharelado Interdisciplinar em Saúde.

\subsection{Instrumento}

O instrumento utilizado para obtenção dos dados foi um questionário semi-aberto. Este foi construído com base em revisão bibliográfica realizada anteriormente (Silva, Sá \& Miranda, 2013; Rios \& Sirino, 2015; Veras et al., 2020), pelo grupo de pesquisa responsável pela investigação, corroborando com as informações obtidas de tais publicações. Foi aplicado primeiramente um piloto com 10 estudantes de medicina e, após as revisões identificadas como necessárias, o instrumento foi reformulado e uma versão final foi aplicada no semestre 2018.1 por 6 pesquisadores do grupo de pesquisa.

Esse questionário foi organizado em três partes: a primeira, contendo 20 questões para coleta de dados referentes às características dos participantes, o que permite a análise por gênero, raça, semestre atual, entre outros; a segunda parte, com 30 assertivas, consistiu em um questionário do tipo Likert de cinco pontos, do "Concordo totalmente" ao "Discordo totalmente", possuindo ponto neutro; e a terceira, constituída por duas questões de múltiplas escolhas e duas questões abertas, de modo que fosse possível analisar os conhecimentos dos estudantes acerca dos assuntos ministrados no eixo e que eles pudessem discorrer sobre a importância do eixo para a sua formação. Assim, para este trabalho, o foco de análise são as respostas dadas à segunda questão aberta da terceira parte do questionário, sendo ela: "O que é um atendimento médico humanizado para você?". 


\subsection{Procedimentos de análise}

O Iramuteq é um software de código fonte aberto utilizado para organização e tratamento de dados textuais ou em formato de matrizes. Ele é desenvolvido na linguagem Python e utiliza o software $R$ em suas análises estatísticas. Ferramentas desse tipo vêm sendo utilizadas no auxílio de investigações qualitativas desde a década de 80 , quando surgiram os primeiros programas com esse fim. Alguns dos benefícios do uso desse software é que ele pode dinamizar algumas etapas do processo metodológico de uma investigação qualitativa, como o auxílio na organização e separação de informações, aumento na eficiência do processo e facilidade na localização dos segmentos de texto e agilidade no processo de codificação, tudo isso além de contribuir positivamente com o rigor metodológico. É importante ressaltar, no entanto, que a análise qualitativa dos dados gerados pelo software depende exclusivamente dos pesquisadores envolvidos no processo investigativo, pois são eles os responsáveis por perceber e atribuir significados aos resultados obtidos. Os principais tipos de análises textuais que esse software permite são: Estatísticas; Especificidades e Análise Fatorial de Correspondência (AFC); Classificação Hierárquica Dependente ou Método de Reinert; Análise de Similitude; e Nuvem de palavras (Camargo \& Justo, 2013; Souza, Wall, Thuler, Lowen \& Peres, 2019).

Considerando que Kelle (2010) afirma que o uso de pacotes de software para análise de corpus textual pode "[...] tornar o processo de pesquisa mais sistemático e explícito, e por isso mais transparente e rigoroso" (p. 408), nesta investigação, optou-se pela Análise Fatorial de Correspondências (AFC) para o tratamento de dados com o Iramuteq. Tudo isso no intuito de identificar possíveis diferenças entre as respostas de estudantes que cursaram - BIS como graduação anterior à entrada em Medicina, em relação aos demais, para os quais este curso é a primeira graduação.

Dessa maneira, inicialmente ocorreu a construção do corpus textual seguindo todas as orientações referentes às normas de formatação quanto a linhas de comando, regras ortográficas e tipo de arquivo (Camargo \& Justo, 2013). A codificação considerou como variáveis ter cursado ou não graduação anterior e o semestre cursado pelo estudante no período da resposta. A isto se sucedeu à etapa de tratamento dos dados no software. Para tanto, optou-se pela AFC como tipo de análise mais pertinente justamente por ela favorecer a comparação entre variáveis. 
A Análise Fatorial de Correspondência permite identificar relações entre variáveis dispostas em um plano cartesiano. O software atribui um valor as palavras do corpus, calcula estatisticamente suas freqüências e correlações e aponta o posicionamento dessas palavras no plano de acordo com o seu grau de correlação. Dessa maneira, o posicionamento dessas palavras em cada quadrante do plano de acordo com as variáveis codificadas, apresenta algum significado na relação de uma delas com as demais. A representação de cada categoria no plano se dá por diversos agrupamentos de palavras ou indivíduos em torno dos eixos, e através de configuração correta dos parâmetros do software, ainda é possível exibir as palavras em tamanhos diferentes proporcionais à intensidade da frequência que foram repetidas no contexto analisado ou também proporcionalmente à intensidade da correlação entre elas de acordo com o cálculo do $X^{2}$ (Camargo \& Justo, 2013).

Matematicamente falando, o centro do plano indica a inércia, e quanto mais distantes desse centro e entre si, mais inversamente proporcional é a relação entre as categorias analisadas. No entanto, tratando-se de análises de agrupamentos de palavras, é importante ressaltar que o fato das variáveis se disporem em pólos opostos não indica precisamente que elas têm sentidos completamente opostos. Na verdade, "um mundo lexical só é possível a partir da existência de outros e essa relação entre os mundos também pode ser de complementaridade" (Nascimento \& Menandro, 2006, p. 78). Então, ainda que os discursos de indivíduos de diferentes categorias possam indicar independência entre si quando essas categorias estão localizadas em pólos opostos do plano cartesiano, pode haver algum grau de relação entre eles visto que discorrem sobre um mesmo assunto, portanto, pertencem a um mesmo universo semântico. A percepção desses detalhes depende da leitura e interpretação dos pesquisadores, reforçando a ideia de que o software auxilia o processo investigativo, mas o caráter qualitativo das análises, ele sozinho não é capaz de contemplar. O tratamento dos dados na AFC gerou um aproveitamento de 78,13\% (percentual acumulado dos eixos - eixo vertical $(30,82 \%)$ e eixo horizontal $(47,31 \%))$ das possíveis relações entre variáveis.

Finalizada a etapa do processamento de dados utilizando a proposta da AFC, sucedeu-se a leitura criteriosa de todas as respostas agrupadas nas categorias pelo software. Essa leitura permitiu a percepção mais clara das aproximações e distanciamentos entre as respostas dos sujeitos da pesquisa e um aprofundamento na leitura dos dados dispostos no gráfico. A partir daí, a discussão dos resultados obtidos se baseou nas informações apontadas pelo programa associadas à reflexão dos pesquisadores diante das falas que compuseram cada categoria e do referencial teórico apropriado. 


\section{RESULTADOS E DISCUSSÃO}

Assumindo como variável analisada o fato de ter cursado alguma graduação anterior ou não, a partir da análise dos resultados obtidos, foram criadas 4 categorias, identificadas por cor no Gráfico 1. Este gráfico evidencia a relação entre as variáveis no plano, apresentando a disposição dos agrupamentos de palavras de acordo com $\mathrm{X}^{2}$ calculado para as categorias. Os termos "classes" e "categorias" são utilizados como sinônimos na apresentação desses resultados.

Cada eixo apresenta um percentual que corresponde à variância das respostas, indicando a presença de diferenças semânticas entre as categorias analisadas. Considerando o percentual individual de cada eixo e relacionando isso à interpretação dos resultados, os eixos e seus pólos podem ser nomeados (Nascimento \& Menandro, 2006). A disposição das classes/categorias em quadrantes diferentes indica justamente que cada uma delas é composta de contextos semânticos específicos, diretamente relacionados ao radical da palavra mais destacada em cada grupo.

Observando o Gráfico 1, através da distribuição das principais palavras de cada categoria sobre os eixos, fica evidente que existe proximidade entre as palavras de todas as categorias no plano fatorial. Ao mesmo tempo, também se verifica uma separação clara dos conceitos principais de cada uma delas. Ademais, reforça-se ainda neste gráfico, que a categoria 1 tem maior dispersão de conceitos em relação às demais e a categoria 4 aponta ser a mais afastada das outras, porque suas palavras-chave demonstram pouca interação com as palavras das outras categorias. Salienta-se que a categoria 1 é a que tem maior representação $(69,5 \%)$ do corpus, seguida da 4 (19,2\%). Logo depois a $2(7,5 \%)$ e por último a categoria $3(3,8 \%)$. É fundamental esclarecer, então, que por apresentarem um aproveitamento do corpus inferior ao de 20 textos (respostas), as categorias 2- Possui graduação anterior em outras áreas, exceto saúde (verde) e 3- Possui graduação anterior em curso da área da saúde (azul); prejudicam a consistência dos dados nesse tipo de análise. Portanto, a discussão dos resultados abordará apenas as categorias 1 - Não possui graduação anterior (vermelho) e 4- Cursou o Bacharelado Interdisciplinar em Saúde (roxo). 


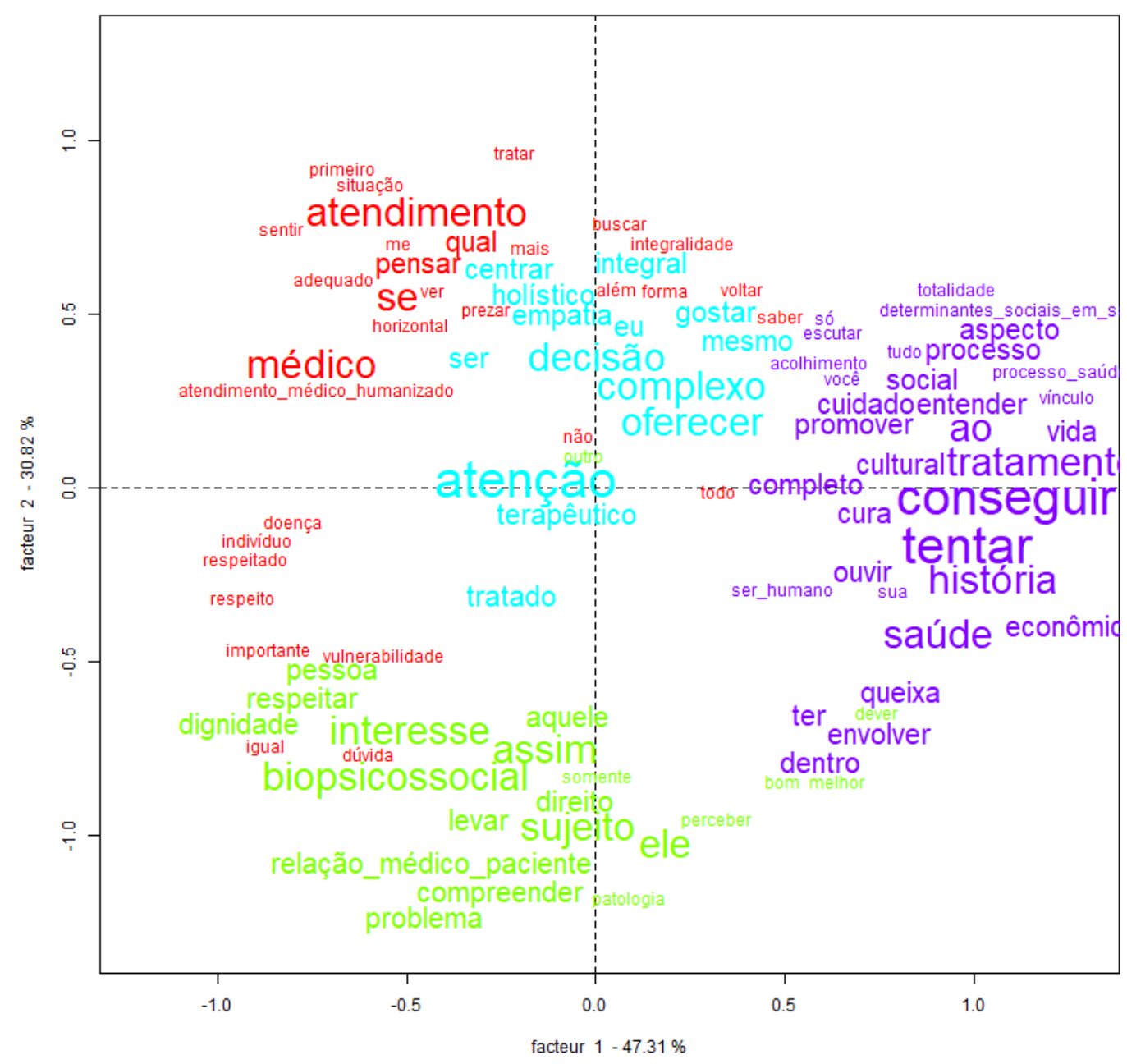

Gráfico 1 - Distribuição dos agrupamentos de palavras de cada categoria de acordo com X² calculado: 1- Não possui graduação anterior (vermelho); 2- Possui graduação anterior em outras áreas, exceto saúde (verde); 3Possui graduação anterior em curso da área da saúde (azul); 4- Cursou o Bacharelado Interdisciplinar em Saúde (roxo).

Historicamente, sabe-se que a dicotomia entre os saberes técnicos e os aspectos humanísticos do fazer médico foi acentuada de acordo com os avanços tecnológicos e científicos incorporados à área da saúde (Reginato, Benedetto, Blasco \& Gallian, 2013). Diante disso, as reflexões acerca da necessidade de se humanizar o atendimento médico se intensificaram a partir da década de 70 e apesar de existirem há um bom tempo, ainda são atuais (Deslandes, 2006). Isto porque tornou-se evidente a necessidade de conciliar os saberes técnicos com os aspectos humanos e éticos, da relação entre profissionais da medicina e seus pacientes (Rios \& Sirino, 2015). 
Dessa maneira, compreender o que estudantes de medicina concebem como atendimento humanizado ajuda a aprofundar esse debate.

As respostas dos estudantes que compuseram as categorias 1 agruparam-se quase que completamente no mesmo quadrante e demonstraram uma predominância de palavras que denotam conceitos e termos importantes da área da saúde, como atendimento, integralidade, atenção, complexo, empatia, horizontal, terapêutico, o que denota percepções de atendimento coerentes com a proposta da Humanização (Deslandes, 2006; Minayo, 2006; Silva, Sá \& Miranda, 2013). Contudo, ao mesmo tempo, ficou clara também uma predominância de ênfase no papel do médico nesse contexto. Como pode ser observado nas respostas dadas à questão "O que é um atendimento médico humanizado para você?":

"É o que consiga de modo integral atender as necessidades do paciente como um todo e não apenas entender certos processos de adoecimento, dando a eles atenção e tentando cumprir da melhor maneira as demandas por ele trazidas". (Estudante $98,1^{\circ}$ semestre)

"É um atendimento com respeito, empatia e consideração ao próximo. Se por no lugar do outro". (Estudante $395,2^{\circ}$ semestre)

É notório, portanto, que os estudantes das categorias 1, em sua maioria, conceberam suas definições de atendimento médico humanizado a partir de uma perspectiva individual, partindo de um olhar direcionado sobre a relação médico-paciente. Entretanto, ficou claro que o foco foi dado ao médico enquanto protagonista sobre as situações, como pode ser observado no fragmento de resposta abaixo:

"É um atendimento onde o profissional consiga estabelecer um vínculo empático com seu paciente, sempre norteado pelo adequado conhecimento técnico-científico, possibilitando uma análise do indivíduo como um todo, dentro dos aspectos biopsicossociais, sempre respeitando suas individualidades". (Estudante $20,8^{\circ}$ semestre)

A grande dispersão das respostas da categoria 1, mencionada anteriormente - e evidente no Gráfico 1, é um ponto a ser ressaltado. Uma hipótese para tal dispersão é que sendo essa categoria a que comportou o maior número de respondentes, ela agrupa muitos estudantes de diferentes semestres do curso, o que pode influenciar na complexidade de suas concepções sobre essa questão. A categoria 4, por sua vez, enfatizou um grupo de palavras que indicam que ao pensarem suas concepções de atendimento médico humanizado, os estudantes demonstraram uma percepção cuidadosa da complexidade do processo saúdedoença, indo além do olhar sobre a relação entre médico e pacientes, mas considerando também os determinantes sociais da saúde. Algumas das palavras mais destacadas foram: 
conseguir, tentar, tratamento, história, saúde, promover, ouvir, cultural, social. Salienta-se, então, a presença de um olhar sobre o atendimento pensado a partir de um ponto de vista coletivo. Isto pode estar relacionado à aproximação maior que o curso do BIS tem com o campo da Saúde Coletiva (Teixeira, Coelho \& Rocha, 2013). As respostas a seguir podem demonstrar:

"A relação médico-paciente perpassa o âmbito do biológico e da cura focada no processo saúdedoença, mas entende o sujeito como um ser biopsicossocial, que está inserido em um contexto social que influencia na sua forma de perceber os processos relativos à saúde. $O$ atendimento médico humanizado é pautado nestas características, e também se baseia no cuidado e no entendimento que o paciente é um indivíduo com autonomia e deve fazer parte das decisões tomadas a seu respeito, como procedimentos, tratamentos e outros. Cabe salientar que a empatia na relação médico-paciente é fundamental para o estabelecimento de vínculos e a quebra de uma relação hierarquizada, colocando assim o paciente como ator principal dentro da perspectiva do atendimento e singularidades do paciente, as quais como raça, estrato social, gênero, cultura, exercendo o saber técnico-científico alinhado à sensibilidade ética ao cuidar." (Estudante 239, $3^{\circ}$ semestre)

"É um atendimento integral, sob a luz de um conceito ampliado de saúde e processo de adoecimento, considerando os Determinantes Sociais em Saúde e olhando o paciente de forma holística, considerando seus aspectos de vida macro sociais e micro sociais e aspectos individuais, aspectos genéticos e estilo de vida." (Estudante $352,2^{\circ}$ semestre)

Funghetto et al (2015) demonstraram que para a construção de um conceito ampliado da saúde na formação dos profissionais desse campo é necessária uma permanente articulação entre as necessidades sociais do Sistema Único de Saúde (SUS) e a educação, buscando suprir estas reais necessidades de atendimento da população. A Humanização dos profissionais e, consequentemente, dos atendimentos, nesse contexto é um dos mais importantes fatores a serem contemplados por esse processo de articulação (Funghetto et al., 2015). A política Nacional de Humanização, inclusive tem papel fundamental nesse cenário por atuar como um dos eixos estruturantes de todas as ações do SUS, tanto no âmbito da formação dos profissionais, quanto do ponto de vista da gestão do sistema e dos serviços propriamente dito (Brasil, 2004).

Diante de tudo isso, é possível formular que na categoria 4 percebe-se se um olhar mais atento sobre o papel do paciente no atendimento. Da mesma forma, a leitura das respostas da categoria permite considerar que a maioria dos indivíduos que a compõem têm concepções de atendimento melhor relacionadas com a proposta da Humanização do que os da categorias 1. Isto porque, eles demonstram perceber essa proposta considerando a 
multifatorialidade que a envolve (Deslandes, 2006). Reforçando essa percepção, a disposição dos agrupamentos de palavras no plano enfatizou também a independência da categoria 4 em relação à categoria 1 . O fato de estarem essencialmente em quadrantes opostos indica que a maioria dos respondentes se polariza sobre os eixos, ainda que haja alguma interação entre suas falas. Essa independência pode ser atribuída justamente ao fato de que ao se pensar o cuidado em saúde e consequentemente o atendimento médico, de maneira mais complexa, não há como atribuir um lugar de protagonista ao médico (Carnut, 2017).

Acredita-se então, que o papel do Bacharelado Interdisciplinar em Saúde (BIS) nesse cenário, foi significativo. Isto porque as respostas demonstram que ter formação no BIS foi um fator determinante para diferenciar a percepção dos estudantes de medicina sobre humanização do atendimento, em relação aos demais, da categoria 1, que não vivenciaram tal experiência, o que expõe também suas noções de saúde, e em especial da complexidade do processo saúde-doença e seus determinantes. Tal fato corrobora, portanto, com um dos objetivos do BIS que é o de possibilitar aos seus estudantes uma compreensão maior do campo da saúde e suas minúcias antes de ingressarem em outra graduação na área.

\section{CONSIDERAÇÕES FINAIS}

Esse estudo procurou analisar se há variação nas concepções dos estudantes de medicina acerca de atendimento médico humanizado entre estudante egressos do modelo de ciclos na UFBA e àqueles que cursam medicina como primeira graduação. Ficou claro na análise que os estudantes egressos do Bacharelado Interdisciplinar em Saúde tendem a conceituar o atendimento humanizado sob uma perspectiva mais ampliada e alinhada com a Política Nacional de Humanização. Cabe acrescentar que se percebeu que eles demonstraram concepções baseadas numa perspectiva de Saúde Coletiva, com uma valorização dos determinantes sociais em saúde. Também foi demonstrado que os estudantes dessa categoria não sustentaram seus discursos enfatizando o processo de adoecimento e sim a partir do conceito ampliado em saúde. Portanto, em que pese o intuito de contribuir para uma formação diferenciada no campo da saúde a partir de uma formação crítica, interdisciplinar e atenta aos problemas atuais, acredita-se que o primeiro ciclo na UFBA contribui para uma concepção de saúde mais ampliada devido à imersão dos estudantes nas humanidades, nas artes e nas ciências. Esse resultado traz consigo a reflexão sobre a 
importância de se valorizar a interdisciplinaridade no currículo médico.

Por se tratar uma pesquisa qualitativa que utilizou questionário com questões abertas, o Iramuteq demonstrou ser um software indispensável para o tipo de análise que se pretendeu neste estudo. O uso desta ferramenta dinamizou o tratamento dos dados e o processo de categorização. A análise fatorial de correspondência demonstrou eficácia na análise das respostas, de modo que ela contribuiu para tornar evidente a diferença entre as respostas de estudantes que cursaram o BIS em detrimento dos demais. Ainda há necessidade de mais estudos que se debrucem sobre a contribuição do BIS no processo de elaboração e/ou amadurecimento da escolha profissional assim como para apreender as contribuições do curso interdisciplinar nas formações tradicionais em saúde.

Agradecimentos. Ao CNPQ pela concessão de bolsa de produtividade à primeira autora e a CAPES pela concessão de bolsa de mestrado à segunda autora.

\section{Referências}

Almeida Filho, N. et al. (2015). Formação médica na UFRB: II O desafio da profissionalização no regime de ciclos. Revista Brasileira de Educação Médica, 39 (1), 123-143.

Barros, N. F. (2014). O ensino das ciências sociais em saúde: entre o aplicado e o teórico. Ciência \& Saúde Coletiva, 19 (4), 1053-1063.

Brasil. (2004). HumanizaSUS: Política Nacional de Humanização: a humanização como eixo norteador das práticas de atenção e gestão em todas as instâncias do SUS. Brasília: Ministério da Saúde, 1-20. Retrievedfrom http://bvsms.saude.gov.br/bvs/publicacoes/humanizasus_2004.pdf

Brasil. (2007). Ministério da Educação. REUNI - Reestruturação e Expansão das Universidades Federais: diretrizes gerais (Portaria n. 552, SESu/MEC, de 25 de junho de 2007). Brasília, DF, 2007.

Camargo, B. V. \& Justo, A. M. (2013) IRAMUTEQ: um software gratuito para análise de dados textuais. Temas Psicol. [online]. 21 (2), 513-518. Retrieved from http://pepsic.bvsalud.org/pdf/tp/v21n2/v21n2a16.pdf

Carnut, L. (2017). Cuidado, integralidade e atenção primária: articulação essencial para refletir sobre o setor saúde no Brasil. Saúde debate [online].41 (115), 1177-1186. Retrieved from http://www.scielo.br/pdf/sdeb/v41n115/0103-1104-sdeb-41-115-1177.pdf

Deslandes, S. F. (2006). Humanização dos cuidados em saúde: conceitos, dilemas e práticas. Rio de Janeiro: FIOCRUZ.

Feuerwerker, L. (2002). Além do discurso de mudança na educação médica: processos e resultados. Rio de Janeiro: Hucitec.

Formigli, V. L. et al. (2010). Projeto político-pedagógico do curso de graduação em medicina da FMB/UFBA. Gazeta Médica da Bahia, 79 (1), 3-47.

Funghetto, S. S. et al. (2015) Perfil profissional tendo o SUS como base das Diretrizes Curriculares da área da saúde no processo avaliativo. Saúde em Redes, 1(3), 103-120. Retrievedfrom http://revista.redeunida.org.br/ojs/index.php/rede-unida/article/view/606/pdf_9

IHAC - Instituto de Humanidades, Artes e Ciências Professor Milton Santos. (2019). Ingresso de estudantes graduados em Bacharelado Interdisciplinar nos Cursos de Progressão Linear da UFBA (Processos 
Seletivos 2012 a 2017). Salvador.

Kelle, U. (2010). Análise com auxílio de computador: codificação e indexação. In: BAUER, M. W.; GASKELL, G. (orgs.), Pesquisa qualitativa com texto: imagem e som: um manual prático; tradução de Pedrinho A. Guareschi. 8. ed. Petrópolis/RJ: Vozes, 393-415.

Kerr, L. R. F. S. \& Kendall, C. (2013). A pesquisa qualitativa em Saúde. Rev rene, 14 (6), 2-4. Retrieved from http://www.periodicos.ufc.br/rene/article/view/3708

Minayo, M. C. S. (2006). Sobre o Humanismo e a Humanização. In DESLANDES, S. F. (org.). Humanização dos cuidados em saúde: conceitos, dilemas e práticas (pp. 23-30). Editora FIOCRUZ, 2006.

Nascimento, A. R. A. \& Menandro, P. R. M. (2006). Análise lexical e análise de conteúdo: uma proposta de utilização conjugada. Estudos e Pesquisas em Psicologia, 6 (2), 72-88. Retrieved from https://www.redalyc.org/pdf/4518/451844612009.pdf

Nery Filho, Antônio et al. (2013). Bioética e literatura: relato de experiência do Eixo ético-humanístico FMBUFBA. Revista Bioética (Impr.), 21 (2), 344-349.

Reginato, V., Benedetto, M. A. C., Blasco, P. G. \& Gallian, D. M. C. (2013) Humanismo: pré--requisito ou aprendizado para ser médico? Revista Brasileira de Medicina, 70 (Especial Oncologia 4), 10--15. Retrieved from https://sobramfa.com.br/wpcontent/uploads/2014/10/2014_jan_humanismo_pre_requisito_ou_aprendizado_para_ser_medico.pdf

Rios, I. C. \& Schraiber, L. (2012). Humanização e Humanidades em Medicina. São Paulo: UNESP.

Rios, I.C. \& Sirino, C.B. (2015). A humanização no ensino de graduação em medicina: o olhar dos estudantes. Revista Brasileira de Educação Médica, 39 (3), 401-409. Retrieved fromhttp://www.scielo.br/pdf/rbem/v39n3/1981-5271-rbem-39-3-0401.pdf.

Rocha, M.N. et al.(2014). Educação Superior em Saúde: contexto institucional de criação do Bacharelado Interdisciplinar. In: Teixeira, C.F. \& Coelho, M.T.A. Interdisciplinaridade na educação superior: o bacharelado interdisciplinar em saúde. Salvador: EDUFBA, pg. 25-42.

Silva, A. M., Sá, M. C. \& Miranda, L. (2013). Concepções de sujeito e autonomia na humanização em saúde: uma revisão bibliográfica das experiências na assistência hospitalar. Saúde Soc., 22(3), 840-852. Retrieved from: http://www.scielo.br/pdf/sausoc/v22n3/17.pdf

Souza, M. A., Wall, M., Thuler, A., Lowen, I., \& Peres, A. (2019). The use of IRAMUTEQ software for data analysis in qualitative research. Revista Da Escola De Enfermagem Da USP, 52, e03353. https://doi.org/10.1590/s1980-220x2017015003353.

Teixeira, C.F.S., Coelho, M.T.C. \& Rocha, M.N.D. (2013). Bacharelado interdisciplinar: uma proposta inovadora na educação superior em saúde no Brasil. Ciência \& Saúde Coletiva, 18(6), 1635-1646. Retrievedfrom: http://www.scielo.br/pdf/csc/v18n6/15.pdf

Traverso-Yépez, M., Morais, N.A. (2004). Ideias e concepções permeando a formação profissional entre estudantes das Ciências da Saúde na UFRN: um olhar da Psicologia Social. Estudos de Psicologia, 9(2), 325-333. Retrievedfrom: http://www.scielo.br/pdf/epsic/v9n2/a14v9n2.pdf.

Veras, R.M, Coelho, M.T.C., Teixeira, C., Traverso-Yépez, M. (2018). A formação em regime de ciclos do Bacharelado Interdisciplinar em Saúde da Universidade Federal da Bahia e a Proposta de Educação Interprofissional. Avaliação, Campinas, 23(2), 294-311.

Veras, RM, Fernandez, CC, Feitosa, CC, Fernandes, S. (2020). Perfil socioeconômico e expectativa de carreira de estudantes de medicina da Universidade Federal da Bahia. Revista Brasileira de Educação Médica, 44(2), e056. 